\title{
Medición de la resiliencia en adultos víctimas del conflicto: Caso Carmen de Bolívar, Colombia ${ }^{1}$
}

\author{
Measurement of resilience in adult victims of the conflict: Case of Carmen de Bolívar, \\ Colombia
}

Alberto Enrique, Candanoza-Henríquez²; Marco Antonio, Rojo-Gutiérrez ${ }^{3}$

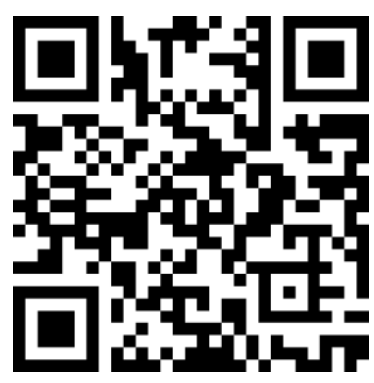

Fecha de recibido: $15-12-2020$

Fecha de aceptado: 29-02-2021

\section{(c)) BY-NC-ND}

Esta obra está bajo una licencia de Creative Commons Reconocimiento-NoComercialSinObraDerivada 4.0 Internacional

\begin{abstract}
RESUMEN
El presente artículo es resultado de una investigación que tuvo por objetivo medir la resiliencia en adultos víctimas del conflicto en el Carmen de Bolívar, Colombia. Para ello, se aplicó un cuestionario estructurado de breve duración, de 32 reactivos donde se evalúan las variables factores protectores internos, factores protectores externos y Empatía. Participaron 231 personas adultas del área geográfica estudiada. Entre los resultados se encontró que entre los factores protectores interno destaca la confianza en si mismos, consideran que la educación es muy importante entre los factores protectores externos y con respecto a la empatía confían en un mejor mañana. Se concluyó que, si bien es limitada la definición y medición de la resiliencia por la naturaleza dinámica y los múltiples factores de influencia del constructo, la población civil encuestada evidencia elementos característicos de la conducta resiliente posterior a haber sido afectados por el conflicto armado en su región.
\end{abstract}

Palabras claves: Adultos, conflicto armado, resiliencia, Carmen de Bolívar.

\begin{abstract}
This article is the result of an investigation that aimed to measure the resilience of adult victims of the conflict in Carmen de Bolívar, Colombia. For this, a short, structured questionnaire of 32 items was applied where the variables internal protective factors, external protective factors and Empathy were evaluated. 156 adults from the geographical area studied participated. Among the results, it was found that among the internal protective factors, self-confidence stands out, they consider that education is very important among the external protective factors and with respect to empathy they trust in a better tomorrow. It was concluded that, although the definition and measurement of resilience is limited due to the dynamic nature and the multiple factors of influence of the construct, the civil population surveyed shows characteristic elements of resilient behavior after having been affected by the armed conflict in its region.
\end{abstract}

Keywords: Adults, armed conflict, Carmen de Bolívar, resilience.

Cómo referenciar este artículo:

Candanoza-Henríquez., A., E., \& Rojo-Gutiérrez., M., A. (2021). Medición de la resiliencia en adultos víctimas del conflicto: Caso Carmen de Bolívar, Colombia. Revista Política Globalidad y Ciudadanía, 7(14), 254-280. https://doi.org/10.29105/pgc7.14-12

\footnotetext{
${ }^{1}$ Este artículo es producto del proyecto de investigación: "El Conflicto Armado En el Municipio El Carmen de Bolívar, Departamento de Bolívar, Colombia: una propuesta de medición de la resiliencia". Iniciado en 2019 y finalizado en 2021.

${ }^{2}$ Institución Universitaria ITSA, Colombia. Magister en Gerencia de sistemas de Gestión Integral por la Universidad Internacional Iberoamericana, Docente Catedrático en la Institución Universitaria ITSA. Email: albertoe.candanoza@ gmail.com. Orcid: https://orcid.org/0000-0002-6555-0797.

${ }^{3}$ Universidad Internacional Iberoamericana, México. Doctor en Estudios Sociales mención Economía Social por Universidad Metropolitana de México. Profesor Investigador en Universidad Internacional Iberoamericana. Email: marco.rojo@unini.edu.mx. Orcid: https://orcid.org/0000-0003-4862-8780
} 


\section{1.- INTRODUCCIÓN}

Ante los episodios de violencia ocurridos en Colombia, frente al tema del conflicto armado, el proceso de paz con la guerrilla de las Fuerza Armada Revolucionaria de Colombia (FARC) y otros actores del conflicto, en la población del Carmen de Bolívar, en el departamento de Bolívar. Resulta de especial interés realizar una medición de la resiliencia, para abordar el fenómeno desde sus diferentes dimensiones, facilitando la identificación de aquellos elementos que permiten desarrollar la capacidad de adaptación y afrontamiento ante situaciones violentas normalizadas en la cotidianidad. A partir de ahí, adoptar medidas que permitan contribuir al mejoramiento de la salud mental de los individuos, que son susceptibles al maltrato, la incapacidad de enfrentar y superar eventos traumáticos.

El presente estudio surge de la necesidad de medir la resiliencia de la población sometida a diferentes sucesos traumáticos, ocasionados por las acciones violentas de los grupos armados al margen de la ley, con el fin de identificar la capacidad que tienen las personas afectadas para convivir con sus victimarios en esta época de post acuerdo. Es así, que la investigación permitirá direccionar la mirada hacia el foco de violencia en Colombia, especialmente en la región del Carmen de Bolívar, de la misma forma se pondrá en evidencia como esta sociedad se ha sobrepuesto a los muchos momentos de adversidad, terror, miedo y pánico generados por estos grupos armados.

Debido a que no se cuenta con suficientes estudios de alcance nacional sobre el fenómeno de la resiliencia, sus estrategias de prevención y actuación con respecto a los acontecimientos causados por el conflicto armado en esa región. El presente trabajo es conveniente desarrollar, para afianzar un mayor conocimiento sobre la ocurrencia de cada tipo de situaciones ante el afrontamiento de esos eventos traumáticos, sus características y las necesidades de intervención. Por otra parte, permite ampliar los datos sobre la resiliencia en estos tipos de sucesos traumáticos, para contrastarlos con otros estudios similares y analizar las posibles variables según el género, nivel socioeconómico, la gestión de las entidades gubernamentales y el contexto.

Así mismo, al ser la propuesta de la resiliencia una mirada que cambia los esfuerzos de los investigadores por analizar la realidad en la medida que centraliza su atención en la población sana a pesar de estar en un medio insano, para lo cual se hace necesario utilizar las herramientas teóricas de las ciencias humanas, tales como: la psicología, las ciencias sociales, la medicina y la política, entre otras. 
En la búsqueda y esclarecimiento de la capacidad que tiene un pueblo de superar la presión de la violencia, para volver a su estado inicial de paz tranquilidad y bienestar humano fortalecido. En este sentido, en la actualidad la resiliencia se reconoce como valiosa, por sus posibles aplicaciones en el área de la salud, la prevención y educación.

Desde la política, por ejemplo: la resiliencia es un tema foco para potencializar a las poblaciones que muestran la fortaleza necesaria para afrontar infortunios que van desde desastres naturales, hasta desastres producidos por actividad antropogénica del hombre como las guerras, así como lo manifiestan algunos autores que han trabajado en resiliencia comunitaria, incrementando el apoyo humano y económico a programas destinados a este objetivo, con estrategias políticas encaminadas a mejorar la calidad de vida de dicha región. Esto implica, que la resiliencia aplicada a las comunidades sea diseñada como una forma de ofrecer afecto y apoyo, para ese grupo de personas que han sido violentadas por los grupos armados al margen de la ley, para que tengan la capacidad de desenvolverse, relacionarse y lo más importante que puedan crear vínculos más cercanos con los demás. Alguien resiliente se caracteriza por ser introspectivo, independiente y por mantener cierta distancia emocional para afrontar las dificultades (Suárez, 2002).

La investigación aporta a las ciencias sociales en general, la percepción de los factores que caracterizan la población en situación de desplazamiento generado por la violencia y los eventos postraumáticos que dejó el conflicto armado en esta región. Un punto de partida en el que se puedan profundizar la comprensión de la resiliencia desde su propia mirada. En la actualidad se viven momentos de crisis complejas, una dinámica de cambios constante por lo cual se debe aprender a convivir con él. Por eso la reconstrucción de resiliencia es uno de los objetivos clave para las iniciativas de transición hacia otros movimientos sociales. Medir la resiliencia permite examinar las capacidades de recuperación ante las fatalidades, conocer las estrategias llevadas a cabo por los individuos para afrontar los riesgos, indagar sobre los factores causantes que explican su desarrollo saludable y finalmente formular estrategias más adecuadas de intervención para el fortalecimiento de las comunidades.

El estudio aporta una unidad metodológica, para que en un futuro se realicen investigaciones que utilicen metodologías compatibles de manera que se posibilitaran análisis conjunto, comparaciones entre períodos temporales concretos y evaluaciones de las intervenciones que se realicen o se estuvieran llevando a cabo para la prevención y tratamiento de la resiliencia, la investigación es viable pues se dispone con los recursos para llevarlo a cabo en la Identificación de los niveles de resiliencia de la 
población estudiada. Es dar un paso en la comprensión de su capacidad de recuperación, luego de los eventos desfavorables a los que han sido expuestos.

El territorio colombiano se ha visto afectado por un conflicto armado interno de más de 60 años, producto de las desigualdades sociales y económicas, según la Unidad para la Atención y la Reparación Integral a las Víctimas (2021), al 01 de enero de 2020 se tienen en el Registro Único de Víctimas -RUV 8.553.416 personas afectadas por algún tipo de violencia en el marco de este conflicto. En el departamento de Bolívar, cuya capital es la ciudad de Cartagena de indias, se registraron hasta la fecha 650.360 personas que suponen la población total del departamento, teniendo un referente de $24 \%$ de personas afectadas en esta región, haciéndose necesario el estudio de diferentes alternativas que permitan a las víctimas superar las situaciones de violencia en el marco de la construcción de paz desde y para los territorios, (Unidad para la Atención y la Reparación Integral a las Víctimas, 2021).

Abordar la resiliencia en al marco del conflicto armado en Colombia, representa crear aportes prácticos que accedan adelantar en el diseño de estrategias que beneficien a las victimas desde un compromiso que retome sus características individuales y sociales, hacia la potenciación de esa participación activa entre el ser y el actuar, donde la pertenencia, la identidad, la motivación, la flexibilidad, la sociabilidad entro otros, crean eco en la reconstrucción individual y social de la resiliencia. Es un esfuerzo más, para crear nuevas incógnitas a la psicología, en particular a la psicología social, a la psicología social comunitaria y a la psicología del desarrollo, de allí su coyuntura disciplinaria.

El beneficio innovador de conocimiento de la presente investigación se halla en el estudio del fenómeno de la violencia desde una perspectiva distinta como es la resiliencia, la cual se centra en descubrir los factores que median el impacto de los factores de riesgos en la salud mental. Y por último esta investigación permitió explorar resultados iniciales, que se convierten en la base para adelantar en el diseño de estrategias de promoción que ayuden a esta población adquirir instrumentos para afrontar situaciones y experiencias dolorosas vividas.

\section{2.- FUNDAMENTO TEÓRICO}

No hay una precisión clara de los inicios sobre el tema de la resiliencia, diferentes autores plantean varios conceptos, pero no hay una exactitud al respeto, pero lo más aportante y es algo que hay que tener en cuenta el aporte de la resiliencia en el campo de la psicología. A la formación del concepto pretendiendo dar a conocer su campo de acción en la psicología, es decir, formas de pensamiento y actuación que lo 
elevan al estatus de constructo en una disciplina. Hoy en día se sabe que la gran importancia que tiene la medición de la resiliencia en casi todos los campos del medio de las ciencias humanas, quien se encarga de analizar el comportamiento del ser en diferentes circunstancias y su forma de reaccionar antes situaciones adversas. La medición de la resiliencia es de gran importancia para el análisis del comportamiento, desde que se estableció este concepto a la psicología se puede decir que existe determinada probabilidad de que algo será o que se practicarán determinadas conductas del individuo ante eventos adversos e implementar estrategias para enfrentarlas (Theis, 2007, p. 78).

\section{Una revisión histórica al concepto de Resiliencia}

Según Ardila (2008) El papel de la psicología para evitar que el círculo vicioso de la violencia continúe (y que vuelvan a aparecer los conflictos, al no haberse solucionado sus causas) puede ser decisivo. La psicología tiene importantes instrumentos conceptuales y prácticos para lograr que los grupos marginados se integren a la sociedad, que se desarrolle un sentido de pertenencia al país, que los colombianos crean en sí mismos y en su futuro. Implica dar empoderamiento a grupos marginados, desarrollar oportunidades laborales, educativas y de ascenso social, autoeficacia, perspectiva de futuro, motivación de logro y de afiliación, etc. (p. 194).

Historialmente el término resiliencia constituye un amplio constructo teórico que tiene lugar en los inicios de los años cuarenta. Desde entonces, es posible vislumbrar un concepto basto y complejo que suscita hoy un gran interés, en quienes trabajan en el campo médico, social, psicológico y educativo. El cual ocasionó un cambio primordial de la perspectiva respecto a la posición pasiva, o solamente receptiva del paciente propio de la década de los 60: "en vez de estudiar las debilidades, las carencias y los medios de compensarlas, se empezó a investigar las fuerzas y cómo usarlas” (Theis, 2007, p. 78). El término comenzó a usarse desde los años cuarenta, pero tuvo su desarrollo y aplicaciones importantes en los años sesenta en el campo educativo, social, médico y psicológico, jugando un papel preponderante en el análisis activo y pasivo de los pacientes utilizando métodos aplicables en cada caso específico

Así mismo Theis (2007), comenta que es importante resaltar la importancia que tiene identificar qué elementos ayudan a superar los problemas y situaciones adversas, esto ¿cómo nos convierte en personas resilientes?, es decir, individuos con capacidad para desenvolverse de manera positiva ante las adversidades. En un principio se pensó que se nacía siendo resiliente, pero después se observó que hay personas que ostentan estas capacidades, y otras no. Entonces, se detectó que se pueden aprender ciertas 
actitudes que lleven a enfrentar positivamente circunstancias adversas. Por lo tanto, el análisis y la identificación de la resiliencia, permite descubrir diferentes elementos para implementar estrategias con el fin de ayudar a los pacientes a enfrentar efectivamente los diferentes episodios traumáticos. Puesto a que, se pensaba que las personas según su genética serian resilientes, pero se llegó a la conclusión que cada individuo reacciona diferentes en cada situación específica y que cada persona puede aprender según sus actitudes a enfrentarlas.

Según Bascon (2012), la resiliencia se refiere a la "capacidad de los sujetos para sobreponerse a periodos de dolor emocional, Sé que un sujeto o grupo de personas manifiestan una resiliencia adecuada cuando pueden sobreponerse a contratiempos o incluso resultar fortalecidos por los mismos” (p. 91).

La resistencia o la invulnerabilidad tiene que ver con la capacidad física que tiene una persona para sobrellevar durante un tiempo una determinada fuerza causada por algunos factores o elementos externos. Si se relaciona con el momento actual, si la fuerza ejercida de este fenómeno pandémico prevalece la capacidad de aguante de los seres humanos, pueden terminar rompiéndose, estableciendo su grado de nivel de la resistencia máxima (Bascon, 2012).

Un aspecto importante del ser humano en el afrontamiento de situaciones críticas para el proceso de recuperación es la resiliencia, que se define como la capacidad que tienen las personas para hacer frente al dolor, a experiencias difíciles o traumáticas (Wilches, 2010). En estas circunstancias, sería la resiliencia la que daría la capacidad a este mismo ser humano de acoplarse y despuntar esta situación del fenómeno pandémico que está sufriendo, y que puede estar en su nivel máximo de resistencia. Esto quiere decir que, el ser resistentes es lo que hace resistir con relación a este medio; una nueva forma de vivir sin llegar a dañarse; y la resiliencia es la capacidad de recuperación cuando ha llegado a romper.

La percepción de estrés elevada se asocia con los niveles de resiliencia, a mayor resiliencia menor nivel de estrés. Las personas que utilizan estrategias de afrontamiento centradas en la tarea manejan mejor el estrés, disminuyendo sus efectos negativos y aumentando el nivel de resiliencia. (Arrogante, Pérez y Aparicio, 2015; García, Salguero, Molinero, De la Vega Ruiz y Márquez, 2015; González y Artuch, 2015; Terzi, 2013).

Según estudios de Mukashema y Mullet (2012), la expresión espontánea y genuina de los sentimientos (tanto de los agresores como de los agraviados) y el conocimiento de los hechos violentos, promueven los sentimientos de perdón y reconciliación en la persona que ha sido víctima. La violencia 
prolongada durante más de 50 años y su progresiva degradación han generado impactos y daños devastadores tanto para las víctimas, familiares, comunidades y organizaciones e instituciones públicas, como para el conjunto de la sociedad colombiana. Generando diversas realidades sociales, donde las víctimas han sentido un abandono por parte del estado.

Como se mostró previamente la resiliencia implementada en el medio del conflicto y la violencia, permite reconocer la capacidad que tienen las víctimas del conflicto armado para superar las dificultades a las cuales han sido sometidas ya sea por grupos al margen de la ley o por acciones gubernamentales. Gracias a esta capacidad, las víctimas logran reconocer los riesgos de su condición (estigmatización, abandono, dependencia) para así adaptarse, recuperarse y reconstruirse gracias a su propia experiencia (Mukashema y Mullet, 2012).

En una denominada segunda generación de estudios, el concepto de resiliencia es ampliado en dos líneas principales a saber: la resiliencia como proceso, y la resiliencia aplicada a programas de intervención social (García y Domínguez, 2013). Este énfasis, nace de la mano con las teorías ecológicas, permitiendo ver el proceso resiliente, como el producto de la interacción entre el micro, meso, exo y macrosistemas (Bronfenbrenner, 1981). Resaltando la importancia del entorno en el que el sujeto está inmerso, y las dinámicas que están en juego, desde el ambiente familiar, hasta la problemática económica del país.

Teniendo en cuenta el apartado anterior, se puede decir que se deben promover acciones para contribuir a la inclusión social de la resiliencia, para que se fructifique la vitalidad, la capacidad y la energía para avanzar prontamente en el presente y construir con el apoyo de un proyecto de vida, se tiene que considerar y tomar acciones y características que promuevan la resiliencia y que este tema debiese ser prioritario no solo para los profesionales de la salud sino para todas aquellas personas que necesiten un seguimiento terapéutico (Bronfenbrenner, 1981; García y Domínguez, 2013).

El dolor emocional y la tristeza son comunes en los individuos que han sufrido grandes fatalidades o traumas en sus vidas. De hecho, el camino hacia la resiliencia seguramente está lleno de impedimentos que pueden afectar nuestro estado emocional. La resiliencia no es una particularidad que la gente tiene o no tiene. Ella incluye conductas, pensamientos y acciones que logran ser aprendidas y desarrolladas por cualquier persona en el transcurso de su vida y en el entorno en que se desenvuelve. La resiliencia, por tanto, se construyó partiendo de las problemáticas de guerra, y de pobreza extrema, que curiosamente 
son las condiciones que prevalecen en el contexto actual de Latinoamérica. Esta revisión del nacimiento del constructo permite avanzar hacia una reflexión más profunda, en la cual se pueda ver de dónde parten las maneras de delimitar la resiliencia (Rodríguez, 2018).

En Latinoamérica debido a las brechas económicas, el contexto para gran parte de los infantes es bastante crítico, generando un alto riesgo estadístico de incidir en la delincuencia, drogadicción, violencia y demás. Kotliarenco, Cáceres y Fontecilla (1997) en su revisión del estado del arte, señalan la importancia que tuvo el cambio de enfoque desde la mirada patológica, que promovía métodos asistencialistas a un enfoque basado en las condiciones que posibilitan un desarrollo más sano del sujeto, y que por lo tanto re-enrutaría la forma en la que se realizaban las intervenciones sociales, que permitan actuar preventivamente contra los efectos nocivos de la pobreza, la violencia, y demás factores de riesgo. Vale la pena aplicar la Resiliencia en la solución de problemas sociales, sobre todo en nuestra región Latinoamericana, la cual no está exenta de tantas dificultades de tipo social político y económico, que han heredado de los primeros colonizadores europeos, es parte de nuestras dificultades, inequidades, desigualdades, la falta de oportunidades, violación de los derechos humanos y tantas vicisitudes.

Es importante de recalcar que, al aplicar la resiliencia en el análisis y solución de problemas sociales, destacar que para cada situación se presentan diferentes situaciones difícilmente repetibles, que acontecen en un momento y contexto específico. Y, por tanto, no se puede generalizar en otros contextos los resultados de una experiencia, dadas las características de cada caso. Así mismo, cada cultura desarrolla sus propias capacidades para enfrentar los problemas y la visión que tiene de ellos, considerando así, que no siempre una misma situación desencadena fatalmente un resultado negativo, sin excluir que una condición difícil aumenta el riesgo de que surja un problema determinado. (Kotliarenco, Cáceres y Fontecilla 1992).

En Colombia el Programa de Resiliencia de FAO, en el período 2017-2020, se inserta en este marco poniendo énfasis en los aspectos de construcción de resiliencia como concepto vertebrador fundamental en los escenarios de pos-acuerdo y de recurrencia de desastres naturales. Así mismo, recomienda que en el país ha sufrido los embates de la violencia de los grupos ilegales, los cuales se han especializado en la extracción de recursos minero-energéticos como oro, carbón, petróleo y ferroníquel, generando un gran impacto ambiental y estas actividades en un contexto de debilidad estatal, han originado conflictos de diferentes dimensiones. Teniendo en cuenta su aproximación conceptual, la resiliencia se convierte en un tema relevante en el trabajo con personas que han sido víctimas del conflicto 
armado, pues permitirá entre otros reconocer las diferentes formas como las poblaciones que han sido expuestas a situaciones de violencia y conflicto. Lo cual, asumen sus nuevas condiciones de vida, de reconocer su potencialidad de reparación, perdón y reconciliación.

Los impactos del conflicto armado interno son complejos, de diverso orden, magnitud y naturaleza. Estos dependen de las características de los eventos violentos sufridos, el tipo de victimario, las modalidades de violencia, las particularidades y los perfiles de las víctimas, su edad, género, etnia, condición de discapacidad, experiencia organizativa, adscripciones políticas y religiosas, el tipo de apoyo recibido, las respuestas sociales frente a los hechos, las víctimas y las acciones u omisiones del Estado. La población civil de las regiones de los Montes de María específicamente de la población del Carmen de Bolívar, fueron afectadas por los enfrentamientos armados producida por grupos armados al margen de la ley, nuestro país aún se sigue estando expuesta a actos de violencia como homicidios, ataques directos, secuestros y reclutamientos forzados. Muchas personas se ven la necesidad de huir y renunciar a sus pertenencias. (Centro Nacional de Memoria Histórica, 2013; Taylor, 2011).

Las acciones que rememoran y enaltecen la memoria de las víctimas y concientizan a la sociedad civil sobre los sucesos ocurridos por el conflicto armado en el país. Asimismo, estas acciones hacen parte de las recopilaciones emprendidas por grupos y organizaciones de personas víctimas de la violencia, líderes, activistas, organizaciones sociales defensoras de Derechos Humanos, y comunidades indígenas y negras seleccionaron una multiplicidad valiosa de información referente a lo sucedido, para que la opinión pública tenga memoria y recuerde los hechos, que sucedieron en medio del conflicto (Centro Nacional de Memoria Histórica, 2013; Taylor, 2011).

En este campo de estudio, hay que considerar que las personas generan estrategias de afrontamiento ante situaciones de amenaza, abordadas desde el campo de la salud mental y de la psicopatología en relación con el estrés, las emociones y la solución de problemas. Las estrategias de afrontamiento se definen como el conjunto de recursos y esfuerzos comportamentales y cognitivos orientados a resolver la situación amenazante o adversa, a reducir la respuesta emocional o a modificar la evaluación que se hace de dicha situación (Lazarus y Folkman, 1986).

Según Lazarus y Folkman (1986), la violencia en Colombia ha sido constante y parece interminable, en este periodo de posconflicto prevalecen los enfrentamientos de los actores de conflicto, la violencia continua está entendida como una condición inherente a las relaciones humanas, también se 
concibe como un problema de salud pública que afecta significativamente la salud mental de las personas implicadas. Algunas de las cifras exponen que hasta un $30 \%$ de las personas expuestas a violencia padecen síndrome de estrés postraumático y depresión, incluso se ha reportado un 100\% en poblaciones con una afectación muy significativa (Larizgoitia et al., 2011).

\section{Resiliencia social}

En este análisis lo que se pretende expresar que algunas características representan un estado ideal de resiliencia- en otras palabras, una comunidad que exhibe todas las singularidades en todos los ejes (temas y componentes) habría alcanzado el nivel de seguridad más alto posible. sin embargo, como el estado ideal de resiliencia siempre estará fuera de nuestro alcance, las organizaciones necesitarán escoger aquellas características que les sean más relevantes a las comunidades, este punto permitirá ampliar dicha medición que se está proponiendo en este trabajo. Por otro lado, se hace necesario tener claro que la resiliencia no solo sirve para resolver cualquier tipo de problema y mejorar las condiciones de bienestar, sino un amplio espectro con respecto a la Vida. Según, la Real Academia Española (2021), este término, proviene del latín y se deriva del verbo resilio, el cual puede interpretarse como volver de un salto o volver atrás. Este vocablo ha conseguido adaptarse con gran contundencia en el mundo de las ciencias sociales y es hoy en día bastante utilizado dentro de la psicología.

Analizando un poco la resultante de las concepciones de resiliencia, es incuestionable la cualidad de ser una capacidad, potencial o pericia. Es una forma de inteligencia a la que se puede llamar, en el marco de este análisis, como inteligencia resiliente. Ésta, es una expresión de la inteligencia total en el ser, que faculta al organismo vivo o persona humana para ubicarse, moverse y trascender los problemas que le traza la existencia, dentro de los escurridos términos vitales que le brinda el contexto, en un momento explícito de su historia vital; esto, gracias a una transformación de conciencia de una forma inferior en dificultades a otra de más alto orden y capacidad para subsistir y trascender. Las investigaciones con enfoque de resiliencia tienen sus orígenes desde los años setenta aproximadamente, inicialmente desde algunos países europeos. Posteriormente en algunos países de américa latina se ha “empezado a gestar la utilización del término en problemáticas endémicas de cada país (Vásquez, 2011).

Existen en Colombia organizaciones de Víctimas del Conflicto Armado, que son las encargadas de la reivindicar a las víctimas de crímenes cometidos por el Estado, sea por acción y omisión en acciones perpetradas por miembros de la fuerza pública, paramilitares u organizaciones al margen de la Ley. Así mismo, propenden por el mejoramiento de la calidad de vida de los integrantes y sus comunidades; 
desempeñan un papel importante a estas comunidades afectadas por este flagelo. Estas organizaciones se congregan de manera estratégica y apoyan a las víctimas con recursos orientados al tratamiento psicológico, para la reconstrucción de su proyecto de vida. A estas instituciones pueden concurrir todas las víctimas en busca de auxilios del Estado y en donde pueden denunciar los hechos por los cuales fueron víctimas de cualquier tipo de conflicto armado en Colombia; en estas, encuentran apoyo emocional y un espacio para expresar su dolor sin caer en la discriminación o la indiferencia.

En Colombia, con base en lo expresado por Vásquez (2011) el Instituto de Bienestar Familiar ICBF, es una entidad pública de carácter descentralizado, es la encargada de proteger los derechos de todos los niños; así mismo trabaja por la prevención y defensa integral de la primera infancia, la niñez, la adolescencia y el bienestar de las familias, a través de la atención integral a niños vulnerables en condiciones de amenaza. Esta entidad cumple la función de restablecer los derechos de los niños y adolescentes, víctimas del conflicto armado que se presenta en la Región de los Montes de María. En las indagaciones que se han realizado sobre trabajos e investigaciones en el tema de resiliencia, existe una gran cantidad de material importante sobre desplazamiento forzado, la violencia generalizada, maltrato, las situaciones de exclusión y precariedad de la población joven en contexto escolar, son temas propios de las problemáticas más frecuentemente exploradas a nivel social y político.

Así mismo, la Real Academia de la Lengua (2016), afirma que resiliencia es: "la capacidad para recuperarse de una adversidad, superar situaciones de dolor y continuar la correspondiente proyección de cara al futuro" (párr. 2); este término se ha convertido en sí mismo en un área de mucho valor e interés, es un paradigma para la investigación y la intervención social, económica, política y psicológica de la región del Carmen de Bolívar. Los seres humanos tienen la capacidad de adaptarse a los cambios, todo de pendiente del factor carácter y personalidad individual y el entorno en que se desarrollan, es por eso por lo que el análisis de la resiliencia y sus resultados en estas circunstancias permitirán abordar la situación para posteriormente realizar las intervenciones correspondientes (RAE 2019).

El conflicto armado interno en Colombia es un conflicto violento en el que se confirma una profunda diferencia cuantitativa y cualitativa entre los recursos militares, políticos y mediáticos de los combatientes comprometidos, y que por lo tanto exige a los bandos a utilizar formas atípicas, que rebasan el manido marco de la tradición militar recurrente. Entre estos medios se cuenta la guerra de guerrillas, la resistencia, toda clase de terrorismo, la contrainsurgencia, el terrorismo de Estado, la guerra sucia o la civil. La Unidad para la Atención y Reparación Integral a las Víctimas (2010), manifiesta que: el Índice 
de Riesgos de Victimización- IRV, asumirá el riesgo de victimización como la posibilidad de ocurrencia de infracciones al Derecho Internacional Humanitario o de violaciones graves y manifiestas a las normas internacionales de derechos humanos, ocurridas con ocasión del conflicto armado interno, en los municipios de Colombia.

El ÍRV es una herramienta importante para el estudio de los diferentes escenarios de victimización en el marco del conflicto armado en Colombia, constituida para monitorear las diferentes causas y efectos, así como para contribuir en la definición de lineamientos de política inclinados a la prevención, protección y garantías de no repetición de tales actos. En la actualidad, algunos países se encuentran afectados por la guerra, esto probablemente se debe a diferentes elementos presentados en esos países; pero, son los civiles, quiénes son la parte más perturbada por estos inconvenientes. Así mismo, Ramírez, Hernández, Romero y Porras (2017), afirman que en el mundo existen 2.7 millones de víctimas, siendo la población adulta la gran mayoría de desplazados por los conflictos armados, debido a los intereses políticos internos de esos países.

La peor parte de las situaciones adversas y traumáticas en los conflictos armados es la población civil quienes directa e indirectamente quedan en medio de los enfrentamientos bélicos, en su mayoría enfrentamientos políticos. A nivel mundial existe una taza de 2.7 millones, en su generalidad personas de la tercera edad (Ramírez et al, 2017). Es importante resaltar que la Organización Panamericana de la Salud (2002), define la violencia como: el uso deliberado de la fuerza física o el poder ya sea en grado de amenaza o efectivo, contra uno mismo, otro sujeto, un grupo o comunidad, que cause o tenga mucha probabilidad de causar lesiones, muerte, daño psicológico, trastornos del desarrollo o privaciones. La violencia es a y sido siempre uno de nuestros mayores problemas que claramente atraviesa como sociedad reproduciéndose en todos los ámbitos de nuestra cotidianidad, manifestándose en todas sus formas.

Todo este tipo de situaciones han venido ocurriendo en nuestro país y no cesaran mientras el estado no haga presencia territorial en regiones apartadas, sin siquiera suplir las necesidades de los pobladores en esos lugares, que la violencia trae consigo, donde imperan otros poderes llamados grupos armados al margen de la ley. Tales como; guerrillas ELN, paramilitares, bandas criminales - BACRIN, narcotraficantes, a esto se suman los disidentes de la FARC, quienes, a pesar de tener un proceso de paz vigente, aun hacen presencia conflictiva.

En Colombia, según el Centro Nacional de Memoria Histórica, (2013), citado por Ramírez et al. (2016), el conflicto armado interno inició en la década de los cincuenta (50) y sigue presente en muchas 
zonas del país, el cual se evidencia en los incesantes enfrentamientos militares de tropas legales e ilegales que ocurren a lo largo de nuestro territorio, el cual ha permitido afectaciones de todo tipo de habitantes. Así, en Colombia existen las dos (2) organizaciones al margen de la ley más longevas de América Latina, como son las Fuerzas Armadas Revolucionarias de Colombia FARC y el Ejército de Liberación Nacional (ELN). Las dos tradicionales guerrillas más antiguas de América Latina, como son las FARC y el ELN, a lo largo de estos tiempos se han sumado como actores del conflicto otro considerable número de grupos armados tales como paramilitares, cárteles de la droga, guerrillas urbanas o de reivindicación indígena, además de bandas criminales las cuales crean pánico y masacres afectando en su gran mayora a la población civil.

Estos conflictos según Ramírez et al (2017), se vienen dando durante este período se han sumado grupos armados como los carteles de narcotraficantes, bandas de criminales, paramilitares, disidentes de la FARC (desertores que no se acogieron a los acuerdos de Paz) organizaciones urbanas al margen de la ley o de reivindicación indígena, las cuales el gobierno ha tratado de erradicar con políticas de dialogo y acciones militares, con el fin de apaciguar muchos episodios violentos. Estas estructuras operan en pequeños grupos dedicados a labores de inteligencia, extorsión, tráfico de armas, propaganda, apoyo a guerrilleros heridos, reclutamiento, ampliación de la organización y diversificación de las fuentes de ingreso el cual es una nueva estrategia urbana del grupo hoy político FARC. La presencia de milicianos infiltrados en las universidades públicas, a sus integrantes se les rotula con la palabra "milicianos" Son hombres y mujeres dedicados a la instrucción política de las comunidades.

Con la frase de Guedán y Ramírez (2005), “Colombia ha sido y es un país abocado a la violencia endémica". Expresan que el conflicto armado en Colombia trasciende desde su inicio independentista, los grandes líderes o también llamados próceres de la independencia entraron en disputas derivadas en los avances de la colonización, reyertas territoriales y la contraposición de sus ideales políticos, la complejidad de los antagonismos partidistas, los conflictos relacionados con la propiedad de las tierras, así el trasfondo de todas estas es prácticamente la debilidad del estado y su incapacidad para dotarse de medios suficientes para ejercer presencia y autoridad en buena parte del territorio nacional. A pesar de que se ha avanzado en ese propósito aún falta mucho por recorrer y la violencia continúa.

Esta enfermedad de la violencia que padece el pueblo colombiano es de vieja data, heredada de la generación criolla, que tomó las riendas de nuestro país desde la independencia, las violaciones de los derechos humanos, la imposibilidad del estado de hacer presencia en lugares inhóspitos, territorios hoy 
ocupados por las organizaciones al margen de la ley que crean violencia para ejercer su poder criminal (Guedán y Ramírez, 2005). La violencia en Colombia es muy compleja y contradictoria en algunos casos incomprensibles, pero de igual forma vislumbran nuevos desafíos y horizontes en materia de seguridad, pero por ahora mientras llegue a su fin esta violencia ha dejado más de 220.000 víctimas mortales, de las cuales 176.000 eran civiles. Aun cuando el estado ha tenido avances con respecto a la seguridad y al fortalecimiento de este, falta mucho por hacer, pasaran años para que en su totalidad se disminuyan los brotes de violencia, esto es un reto, por ahora el aumento de las víctimas por el posconflicto prevalecen (Ramos, 2017).

Con relación al tema de las víctimas, según el Congreso de la República (2011), en su Ley 1448 de 2011, se consideran víctimas: Aquellas personas que individual o colectivamente hayan sufrido un daño por hechos ocurridos a partir del $1^{\circ}$ de enero de 1985, como consecuencia de infracciones al Derecho Internacional Humanitario o de violaciones graves y manifiestas a las normas internacionales de Derechos Humanos, ocurridas con ocasión del conflicto armado interno. De este modo, ha sido esta misma guerra la que ha arruinado los sueños de miles de personas, que entre las balas, se pierden sus ilusiones, sus sueños y sus expectativas de vida se disuelven y esfuman, como el humo originado por un arma de fuego en una noche violenta de algún campo colombiano, azotado por las balas, la muerte y lo peor, "la incapacidad e impotencia del Estado" que se evidencia en la cada vez más creciente población en situación de desplazamiento forzoso que en ciudades capitales de nuestro país.

A su vez, se podría afirmar que las vicisitudes a los que han sido sometido las víctimas en Colombia, con base en lo expresado por el Congreso de la República (2013), en su Ley 1448 de 2011 , están los homicidios realizados contra algunas comunidades para generar los desalojos y la perdida de sus tierras, a través de tortura, tratos crueles, brutales, indignos, sanguinarios, por medio de diferentes tipos de secuestros y extorsiones; también la desaparición forzosa, las tomas armadas de otras poblaciones, las matanzas indiscriminadas de civiles, el retiro obligado masivo de familias, los motines , las masacres y muertes, delitos contra la libertad, la integridad sexual y la violencia de género, las minas antipersonales, el reclutamiento de forzado de menores, y el despojo de sus tierras.

Estos hechos victimizantes según el Centro Nacional de Memoria Histórica (2013) citado por Ramírez et al. (2017), "se clasifican como delitos contra la vida, la libertad y la integridad, lo que ha conllevado a la crisis humanitaria y de derechos humanos, en varias regiones del país" (p.3). Por otra parte, los resultados del conflicto armado interno en Colombia son muy complicados, y de diversa 
naturaleza. Estos resultados dependen de las peculiaridades de los sucesos violentos generados, tales como: el tipo de victimario, las características de violencia, las peculiaridades y los aspectos de las víctimas, tales como: edad, género, etnia, situación de discapacidad, organización, posición política y religiosa, si ha recibido apoyo del gobierno, y del Estado (Ramírez, 2016).

\section{Medición de la Resiliencia}

Resulta prioritario identificar las posibles formas de medición y evaluación de este constructo, con la finalidad de medir el impacto y la eficacia de los programas de intervención. En tal sentido, en la actualidad, se disponen de varios métodos para lograrlo. Al respecto Luthar y Cushing (1999) definen la Resiliencia como "un proceso dinámico que tiene como resultado la adaptación positiva en contextos de gran adversidad" (p.543). Esta definición enmarca tres mecanismos fundamentales que deben estar presentes en el concepto de Resiliencia: 1. La noción de adversidad o amenaza al desarrollo humano, 2. La adaptación positiva de la adversidad, y 3. El proceso que considera la dinámica entre mecanismos emocionales, cognitivos y socioculturales. En resumen, las distintas definiciones de Resiliencia subrayan en características de los sujetos tales como: habilidad, adaptabilidad, baja susceptibilidad, enfrentamiento efectivo, capacidad, competencia, resistencia a la destrucción, conductas vitales positivas, temperamento especial y habilidades cognitivas, todas desarrolladas frente a circunstancias estresantes que les permiten superarlas.

Aplicada en el entorno del conflicto y la violencia, la resiliencia se puede explicar, como la capacidad que tienen las comunidades para vencer las dificultades a las cuales han sido doblegados ya sea por grupos al margen de la ley o por acciones gubernamentales. Es importante resaltar el efecto que produce las situaciones adversas en los seres humanos, la resiliencia es una herramienta que todos los seres vivos tienen a su disposición, aunque de formas diferentes. Cada persona por razones netamente fisiológicas e individuales manifiesta formas diferentes de adaptación y de enfrentar situaciones conflictivas (Luthar y Cushing 1999).

Según Del Solar (2020), nunca se debe dejar de intentar en la resiliencia, porque hay innumerables casos de éxito. No obstante, cabe resaltar que en situaciones extremas se genera una energía única y especial, que, canalizada correctamente, puede ser de gran ayuda para adaptarse y superar de manera eficiente situaciones similares. Esto permite discernir en la importancia que se asume analizar la resiliencia en el contexto cultural, otro motivo importante de análisis que como punto de investigación 
se podría considerar más adelante en este estudio, es el aspecto cultural como base de los procesos de resiliencia.

También se destaca que la Resiliencia se desarrolla en función de procesos sociales e intrapsíquicos; Generalmente se refiere al conflicto surgido entre dos tendencias opuestas dentro de uno mismo. Está en función de los procesos interaccionales entre las personas y estos, a su vez, contribuyen a la construcción del sistema intrapsíquico del ser humano. han realizado una descripción de ellos, los cuales se detallan a continuación:

\section{Medición de adversidad}

A través de este tipo de medición es posible diferenciar tres formas diferentes de medir la adversidad o el riesgo:

\section{Medición de riesgo a través de múltiples factores}

Este tipo de medición se caracteriza por medir diferentes factores en un solo instrumento. Generalmente el instrumento es una escala de eventos de vida negativos donde el individuo debe especificar aquellos eventos que han estado presentes, como, por ejemplo: Separación de los padres, enfermedad mental de los padres, experiencia de guerra, entre otros.

Situaciones de vida específica. La naturaleza del riesgo está determinada por aquello que la sociedad, los individuos o los investigadores han considerado una situación de vida estresante. Por ejemplo, la experiencia de un desastre natural o la muerte de un familiar. Ambas situaciones han demostrado afectar al individuo haciéndole más vulnerable al estrés, por lo tanto, más vulnerable a otros factores de riesgo.

La dificultad de considerar situaciones de vida como eventos estresantes para medir resiliencia, consiste en discriminar aquellos factores que tienen directa relación con el riesgo (factores proximales) versus aquellos factores que pueden estar mediando entre el riesgo y el resultado esperado (factores distales).

Constelación de múltiples riesgos. Refleja las complejidades del mundo real ya que considera la interacción entre los factores provenientes de los niveles: Social, comunitario, familiar e individual simultáneamente y cómo esta interacción influencia el desarrollo humano y la superación de la 
adversidad. La constelación de múltiples riesgos es un mapeo de las fuentes de adversidad a las cuales se les asigna un puntaje determinado.

\section{Medición de adaptación positiva}

En este tipo de medición, las estrategias son similares a las utilizadas para medir la adversidad, lo que cambia es la forma en que se operacionaliza el constructo.

Adaptación según factores múltiples. Este método mide la adaptación en base al logro de metas de acuerdo con la etapa del desarrollo de la persona. Se dice que son factores múltiples ya que se les pregunta a los profesores, padres y amigos, además de una prueba específica para medir la conducta que va a determinar la adaptación positiva. En cada comunidad el foco de lo que se definirá como adaptación positiva varía, pero lo importante es que está relacionado con la definición de riesgo.

Ausencia de desajuste. Este tipo de medición se utiliza en investigaciones de resiliencia en personas con serios riesgos de psicopatología. Los instrumentos utilizados son generalmente cuestionarios clínicos para identificar desórdenes psiquiátricos. Aun cuando generalmente la resiliencia es asociada con la presencia de competencia a pesar de la adversidad, en este caso, el énfasis está en la superación de la adversidad y el no presentar sintomatología. Esta forma de medición es restringida a aquellos casos de extremo riesgo y generalmente en el área clínica.

\section{Constelación de adaptación:}

Es otra metodología de medición de la adaptación positiva que está basada en diferentes conductas o tipos de adaptación. Los índices elegidos tienen directa relación con el modelo teórico, que generalmente se basan en teorías del desarrollo. Este método incorpora puntajes de pruebas y escalas, más opiniones de otras personas. Por ejemplo, una adaptación positiva en la escuela se mide en función de la obtención de buenas calificaciones y adecuada disciplina en la clase, además de entrevistas a compañeros y padres.

\section{Medición del proceso de resiliencia}

En este caso se refiere a la unión crítica entre adversidad y adaptación positiva. Los dos modelos que intentan evaluar el proceso son: 
Modelos basados en variables. Parte de un análisis estadístico que indaga las conexiones entre variables de riesgo o adversidad, resultados esperados y factores protectores que pueden compensar o proteger los efectos de riesgo. Este modelo permite replicar aquellos patrones o interacciones que demostraron ser exitosos en la adaptación resiliente.

Modelo basado en individuos. Esta forma de medición compara individuos a lo largo del tiempo. Se pregunta que diferencia a niños resilientes de niños no resilientes a lo largo de su vida o en alguna área en particular. Este enfoque pretende capturar interacciones entre factores que ocurren en forma natural y plantear hipótesis respecto a qué fue lo que causó la diferencia en el resultado de la adaptación.

Después de haber examinado los diferentes métodos para medir la resiliencia, es necesario revisar algunos de los instrumentos disponibles que existen en la comunidad científica.

\section{Resiliencia y Vulnerabilidad: medición de la vulnerabilidad.}

Es importante afirmar que se entra en controversia cuando se cuestiona la relación entre resiliencia y vulnerabilidad como sujeto a la medición la vulnerabilidad pone el acento en la sensibilidad a las perturbaciones, mientras que la resiliencia se ocupa de las diversas maneras en que un sujeto dado se prepara y responde a los escenarios de crisis y factores de estrés que amenazan su calidad de vida. (Adger, 2006; Folke, 2006).

El hombre es vulnerable a las armas de fuego o a la potencia de un tsunami. A los desastres naturales, a los actos violentos, Sin embargo, al utilizar la dimensión "vulnerabilidad-invulnerabilidad" se convierte en una característica gradual. Todos los seres humanos son vulnerables ante un virus, pero las personas que tienen menos defensas en su organismo tienen más vulnerabilidad ante la infección. Se trata siempre de la relación entre un organismo y otro elemento, entre el sujeto y una situación. Los sujetos hipersensibles van a percibir las estimulaciones excesivas de su entorno como agresiones dolorosas. Esa vulnerabilidad se aplica también a la sensación de miedo o fobias cada persona tiene deferentes formas de percibir el miedo ante todo hay predisposiciones de eventos ocurridos en el pasado el cual queda fijado en el inconsciente. (Adger, 2006; Folke, 2006). 


\section{3.-MÉTODO}

\section{Diseño}

Esta investigación responde a un diseño de tipo no experimental, ya que no se pretende manipular deliberadamente las variables independientes; es decir, se realiza una observación de situaciones ya existentes. En este caso, la variable violencia, situación que ya ha ocurrido y no es posible manipularla. El diseño no experimental observa fenómenos tal y como se dan en su contexto natural; no hay estímulos a los cuales se exponga a los sujetos de estudio, dada la incapacidad de influir sobre las variables y sus efectos (Hernández, Fernández y Baptista, 2017; Candanoza y Lechuga, 2015).

Según la recolección de los datos de la presente investigación, el diseño adecuado para este estudio es transaccional- correlacional con el fin de describir relaciones entre las variables. En esta investigación, como se dijo, la relación será la capacidad de resiliencia que tiene la población para afrontar eventos catastróficos relacionados con el conflicto armado en Colombia específicamente el municipio del Carmen, departamento de Bolívar. La base epistemológica de la investigación es el enfoque empírico analítico que se basa en la percepción directa del objeto de investigación y del problema (Hernández et al., 2017).

La investigación que en estos momentos está en curso se considera de corte mixto, porque sus fundamentos están enfocados en analizar la resiliencia de la sociedad colombiana ante el conflicto armado de las FARC y la firma de acuerdos de paz: el estudio de caso del municipio el Carmen, departamento de Bolívar, las investigaciones de tipo mixto se constituyen, día a día, en una excelente alternativa para abordar temáticas de investigación en el campo educativo (Hernández et al., 2017).

\section{Instrumentos}

El cuestionario de resiliencia para adolescentes y adultos (González y Valdez, 2010) es un instrumento de autoinforme que consta de 32 reactivos o preguntas, de opciones de respuesta tipo Likert, dividida en tres dimensiones: factores protectores internos (14 reactivos), factores protectores externos (11 reactivos) y factores de empatía (7 reactivos). La aplicación del instrumento se realizó de forma individual, con un límite de tiempo aproximado de 20 a 30 minutos. La instrucción consistió en colocar solo una X como respuesta por cada afirmación de acuerdo con lo que considere de sí mismo. El cuestionario fue calificado a partir de los siguientes valores que cada una de las opciones de respuesta tiene: 
El valor de la opción Siempre es cinco (5), el valor de la opción, la mayoría de las veces es cuatro (4), el valor de la opción Indeciso es tres (3), el valor de la opción Algunas veces es (2), y el valor de la opción Nunca es (1).

Los Factores protectores internos se identifican en los ítems: 17, 18, 20, 21, 24, 23, 25, 26, 27 , 28, 29, 30, 31 y 32. Total reactivos $=14$. Los Factores protectores externos se identifican en los ítems: $1,4,5,6,10,11,12,13,14,15$ y16. Total, reactivos $=11$.

Los Factores de Empatía se identifican en los ítems: 2, 3, 7, 8, 9, 19 y 22. Total reactivos $=7$. Para obtener el valor para cada factor, se suma el valor de cada reactivo; el resultado de la suma total de los valores en cada factor se analiza en acuerdo de los siguientes niveles. Para conocer el nivel de resiliencia del individuo.

Puntuación total para establecer el nivel resiliencia: mínima 32, máxima 160. De los cuales corresponde por indicación estricta del instrumento los siguientes niveles: resiliencia baja de 32 a 74 puntos, resiliencia moderada de 75 a 117 puntos y resiliencia alta de 118 a 160 puntos. En el análisis de los resultados se presentan los niveles obtenidos para cada factor.

\section{Participantes}

Se aplicó el cuestionario a 231 individuos del municipio del Carmen de Bolívar, departamento de Bolívar en Colombia, contactándose con ellos por vía telefónica, y redes sociales teniendo en cuenta el cuestionario de resiliencia para adolescentes y adultos (González y Valdez, 2010). Los datos fueron registrados y procesados a través del software SurveyMonkey.

\section{Tabla 6.}

Ficha técnica de la investigación

\begin{tabular}{ll}
\hline Universo & Adultos habitantes de El Carmen de Bolívar \\
Ámbito & Regional (El Carmen de Bolívar, Colombia) \\
Método de recogida de información & \\
Unidad muestral & Adulto afectado por el conflicto armado residente del Carmen de Bolívar \\
Tamaño de la muestra & 231 \\
Muestreo & No probabilístico de conveniencia \\
Trabajo de campo & abril de 2021 \\
Número de encuestas realizadas & 231 \\
Respuestas válidas & 231 \\
\hline
\end{tabular}


Fuente: Elaboración propia (2021).

\section{4.- RESULTADOS}

\section{Factores protectores internos}

El $82.53 \%$ de las respuestas dadas por los encuestados fue positiva, el $6.19 \%$ fueron neutras y el $11.27 \%$ fueron negativas. Como se muestra en el grafico el $46.63 \%$ de los encuestados en este factor están siempre presto a brindar lo mejor de sí ante las situaciones que se le presentan en el camino. Según Patterson (1988), los factores protectores pueden considerarse como los recursos que tiene una familia para enfrentar su desarrollo y eventos vitales. Son características, rasgos, habilidades, competencias, medios, tanto de los individuos de una familia, de la familia como unidad y de su comunidad. En este caso se analizaron los indicadores factores protectores internos los cuales permiten analizar o hacen refieren a atributos de la propia persona: estima, seguridad y confianza de sí mismo, facilidad para comunicarse.

Ilustración 1.

Factores protectores internos

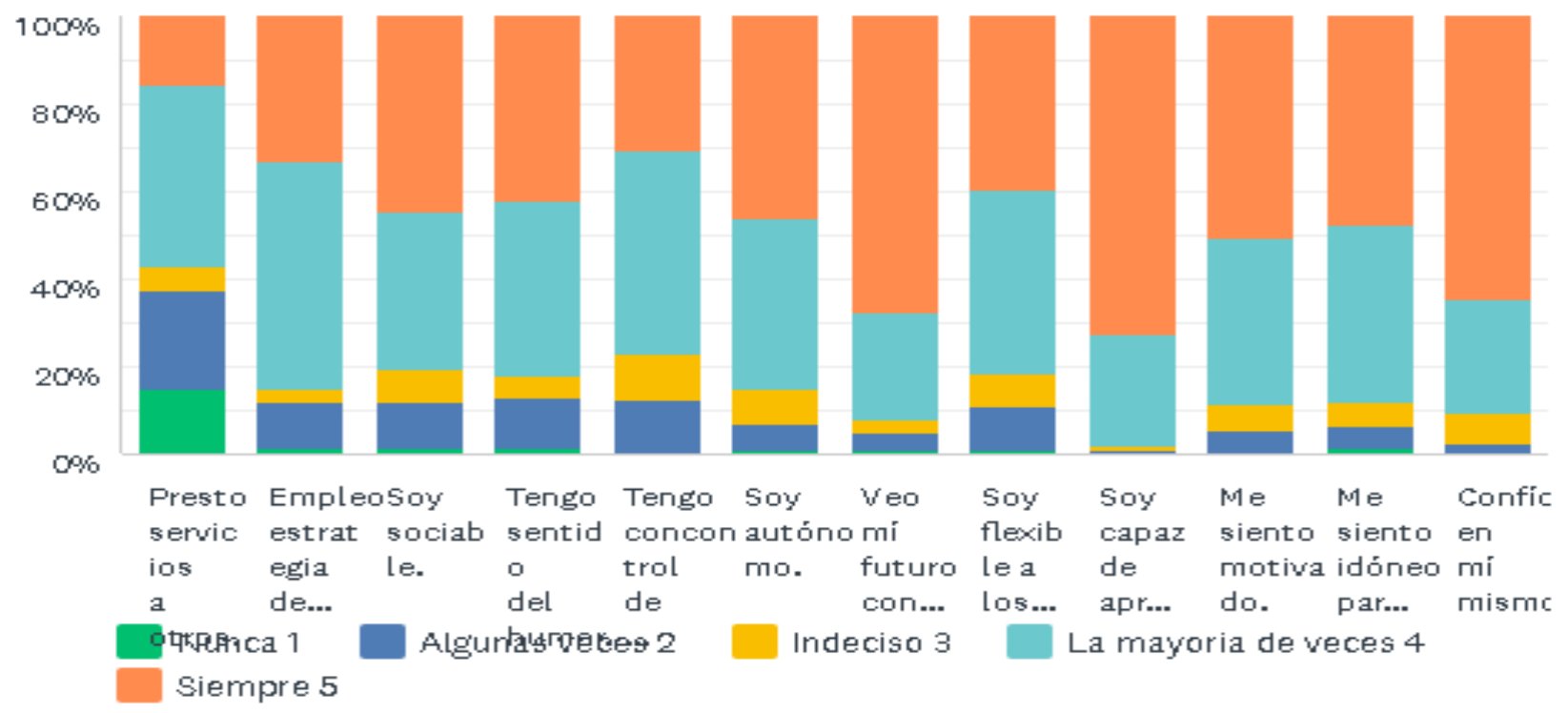

Fuente: Elaboración propia (2021).

\section{Factores protectores externos}


El $85.96 \%$ de las respuestas dadas por los encuestados fueron positivas, el $5.26 \%$ fueron neutras y el $8.76 \%$ fueron negativos. En este factor se muestra que apresar que las personas están siempre con la oportunidad de dar lo mejor de sí mismo, también es de mucha relevancia que en un porcentaje alto la respuesta la mayoría de las veces aumentó en comparación a los factores protectores internos. Los factores externos se refieren a condiciones del medio que actúan reduciendo la probabilidad de daños: familia extendida, apoyo de un adulto significativo, o integración social y laboral.

\section{Ilustración 2.}

Factores protectores externos

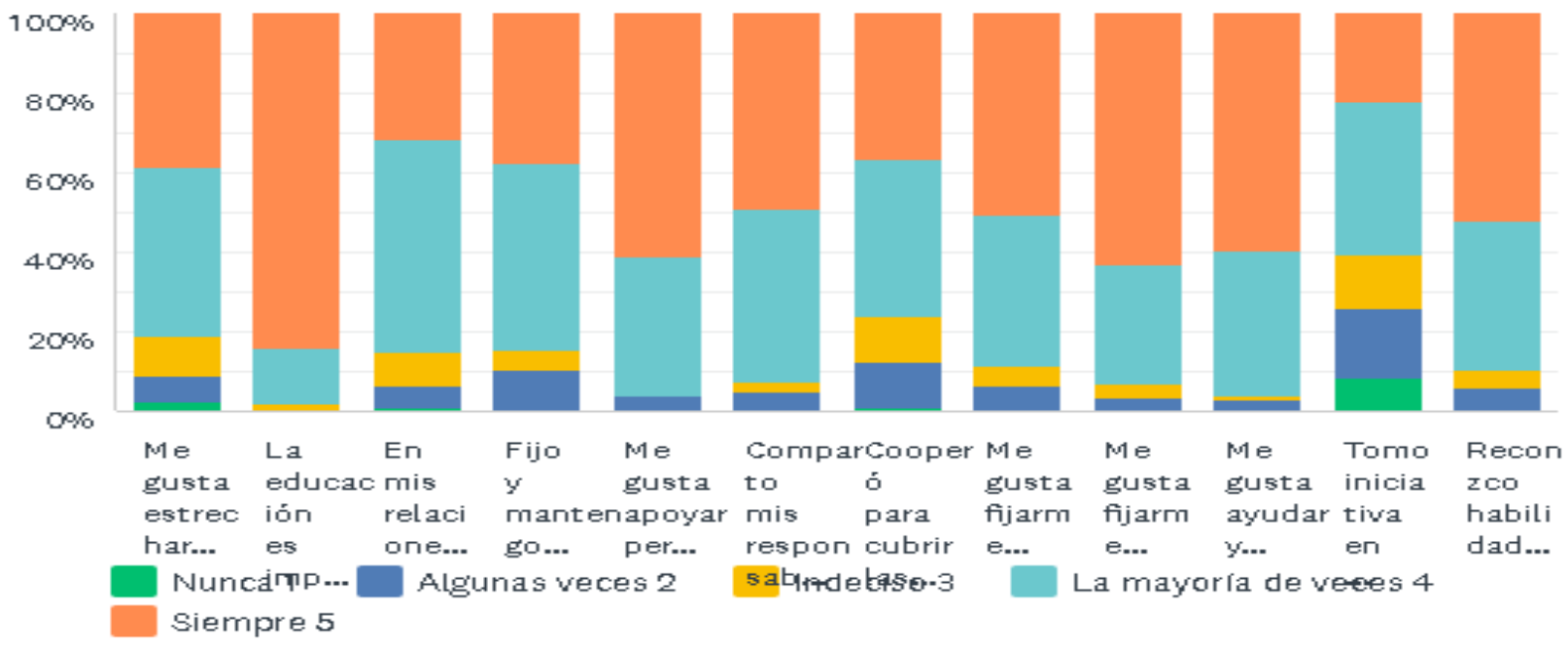

Fuente: Elaboración propia, (2020).

\section{Empatía}

El $86.67 \%$ de las respuestas dadas por los encuestados fueron positivas, el $5.92 \%$ fueron neutras y el $7.51 \%$ fueron negativas. Se puede afirmar que las personas en su gran mayoría en un 75\% tienen mejores expectativas al futuro y tienen la confianza de que habrá un mejor mañana.

\section{Ilustración 3.}

\section{Empatía}




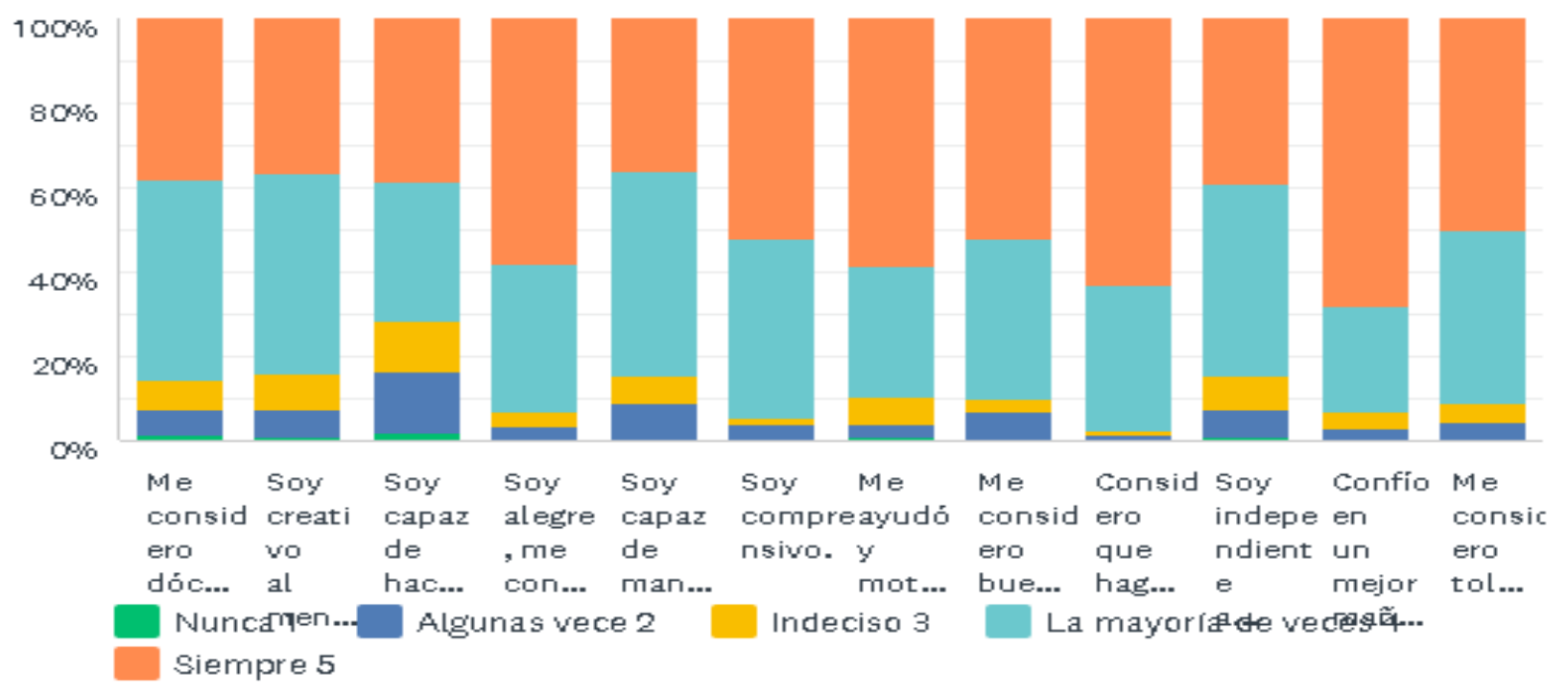

Fuente: Elaboración propia, (2020).

\section{5.- CONCLUSIONES}

En los apartados anteriores se ha podido comprender la resiliencia en adultos víctimas del conflicto en el Carmen de Bolívar, Colombia. En definitiva, sobre la base de los resultados obtenidos con la realización de las encuestas, se pueden enumerar algunas conclusiones generales:

Las victimas que padecieron violencia sociopolítica tienen mayor probabilidad de sufrir trastornos, por tanto, el impacto de la violencia es significativo y requiere de un acompañamiento psicosocial para el restablecimiento de sus derechos humanos.

En Colombia, el uso de la violencia y la lucha por el poder, inicialmente justificadas por a desigual repartición de la tierra y la falta de espacios para participación política, han marcado las dinámicas sociales y políticas, atenuadas por narcotráfico, el narcoterrorismo, la presencia de nuevos actores políticos y armados en un contexto de lucha revolucionaria.

En este sentido, la población habitante del Carmen de Bolivar, se convirtió con el paso del tiempo en un grupo humano vulnerable por el conflicto armado en la región, entendiendo la vulnerabilidad como el estado de susceptibilidad al daño por exposición a tensiones asociadas con el cambio ambiental y social y por la ausencia de capacidad de adaptación. 
Así, las víctimas del conflicto armado requieren de un proceso de perdón y reconciliación que implica reemplazar las emociones negativas hacia un agresor por emociones positivas y olvidar el agravio. Allí aparece el constructor de la resiliencia, como un proceso en el que, a pesar de todos los pronósticos negativos, hay una reinvención del sujeto que se erige por encima de las extraordinarias circunstancias, el fenómeno rebasa la capacidad predictiva de las ciencias exactas y por tanto se requiere de una comprensión del constructo desde una mirada crítica y compleja, que aborde al sujeto desde una mirada trans e interdisciplinar.

Finalmente, se puede afirmar que la presente investigación sirve como paso previo para futuras líneas de investigación, que incluyan una investigación empírica encaminada a profundizar en el conocimiento de otros factores protectores que influyen en la resiliencia. Siendo consciente de ello se considera oportuno dar continuidad a este estudio planteando como futuras líneas de trabajo el desarrollo de una investigación empírica sobre otras mediciones de la resiliencia, evaluación de la resiliencia en otras regiones geográficas y evaluación de la resiliencia en otros grupos etarios (Lechuga, 2019).

\section{REFERENCIAS}

Adger, W. N. (2006). Vulnerability. Global Environmental Change, 16 (3), 268-281.

Ardila Ardila, R. (2008). Psicología de la paz. el papel de la psicología en la reconciliación nacional. Psychologia. Avances de la disciplina, 2 (1), 193-199.

Arrogante, O.; Pérez, A. y Aparicio, E. (2015). Bienestar psicológico en enfermería: relaciones con resiliencia y afrontamiento. Enfermería Clínica, 25 (2), 73-80.

Bascón Díaz, M. (2013). Conflictividad y Violencia de Género en Adolescentes. Un Estudio Discursivo del Ajuste Psicológico en Escenarios Socioculturales. Conflicto, género y ajuste psicosocial en adolescentes. Prisma Social, 11, 260-293.

Bronfenbrenner, U. (1981). The Ecology of Human Development: Experiments by Nature and Design. Harvard University Press.

Candanoza Henríquez, A. E. y Lechuga Cardozo, J. I. (2015). Caracterización de la satisfacción laboral y condiciones de trabajo de una empresa de seguridad en la ciudad de Barranquilla durante el año 2012. Revista Colombiana Salud Libre, 10(2), 98-102. https://doi.org/10.18041/19007841/rcslibre.2015v10n2.1427 
Candanoza-Henríquez., A., E., \& Rojo-Gutiérrez., M., A.

Centro Nacional de Memoria Histórica (2013). ¡Basta ya! Colombia: memorias de guerra y dignidad. Autor.http://centrodememoriahistorica.gov.co/descargas/informes2013/bastaYa/capitulos/bastaya-cap2_110-195.pdf

Congreso de la Repúnlica (2011). Ley 1448 de 2011. Por la cual se dictan medidas de atención, asistencia y reparación integral a las víctimas del conflicto armado interno y se dictan otras disposiciones. http://www.secretariasenado.gov.co/senado/basedoc/ley_1448_2011.html

Folke, C. (2006). Resilience: The emergence of a perspective for social-ecological systems analyses. Global Environmental Change,16 (3), 253-267.

García, X., Salguero, A., Molinero, O., De la Vega, R., Ruiz, R. y Márquez, S. (2015). El papel del perfil resiliente y las estrategias de afrontamiento sobre el estrés-recuperación del deportista de competición. Kronos, 14(1), 1-13.

García-Vesga, M.C. y Domínguez, E. (2013). Desarrollo teórico de la Resiliencia y su aplicación en situaciones adversas: Una revisión analítica. Revista Latinoamericana de Ciencias Sociales, Niñez y Juventud, 11(1), 63-77.

González, M.C. y Arturch, R. (2015). Perfiles de resiliencia y estrategias de afrontamiento en la universidad: variables contextuales y demográficas. Electronic Journal of Research in Educational Psychology, 12(3), 621-648.

González-Arratia, N. y Valdez Medina, J. (2015). Resiliencia. Diferencias por Edad en Hombres y Mujeres Mexicanos. Acta de Investigación Psicológica - Psychological Research Records, 5 (2), 1996-2011.

Guedán, M. y Ramírez, R. (2005). Resolución de conflictos en el S. XXI. CICODE.

Hernández, R.; Fernández,C. y Baptista, P. (2017). Metodología de la investigación. Mc Graw Hill.

Kotliarenco, M.; Cáceres, I. y Fontecilla, M. (1992). Estado de Arte en Resiliencia. Organización Panamericana de la Salud. http://www.ugr.es/ javera/pdf/2-3-resiliencia\%20libro.pdf

Larizgoitia, I., Izarzugaza, I., Iraurgi, I., Ballesteros, J., Forero, C., Markez, I., y Alonso, J. (2011). Impacto de la violencia colectiva en la salud. Resultados del estudio ISAVIC en el País Vasco. Gaceta Sanitaria, 25(2), 108-114.

Lazarus, R. S. y Folkman, S. (1984). El concepto de afrontamiento en estrés y procesos cognitivos. Martínez Roca

Lazarus, R. S. y Folkman, S. (1986). Estrés y procesos cognitivos. Martínez Roca.

Lechuga-Cardozo, J. I. (2019). Estrategia de responsabilidad social empresarial de una terminal portuaria en Barranquilla, Colombia. Signos, 11 (1), 2021, 131-144 
Luthar, S. y Cushing, G. (1999). The construct of resilience: Implications for interventions and social policy. Development and Psychopathology, 26(2), 353-372.

Mukashema, I. y Mullet, E. (2012). Unconditional forgiveness, reconciliation sentiment, and mental health among victims of genocide in Rwanda. Social Indicators Research, 113 (1), 121-132.

Organización Panamericana de la Salud (2002). Informe mundial sobre la violencia y la salud: Resumen. Autor. https://www.mscbs.gob.es/ciudadanos/violencia/docs/informeOMS.pdf

Patterson, J. (1988). Families experiencing stress: The family adjustment and adaptation response model. Family Systems Medicine, 5(2), 202-237.

Ramírez-Giraldo, A., Hernández-Bustamante, O., Romero-Acosta, K. y Porrras-Mendoza, E. (2017). Estado de salud mental de personas víctimas del conflicto armado en Chengue. Psicología desde el Caribe, 34(1), 1-20.

Ramos Vidal, I. (2018). Desplazamiento forzado y adaptación al contexto de destino: el caso de Barranquilla. Perfiles latinoamericanos, 26 (51), 301-328. 10.18504/pl2651-012-2018

Real Academia Epañola (2021). Resilencia. https://dle.rae.es/resiliencia

Rodríguez, D. (2018). Una revisión al constructo resiliencia. Historia y panorama actual. (Trabajo de grado Psicología, Universidad de San Buenaventura Colombia, Santiago de Cali). Archivo digital.

http://bibliotecadigital.usb.edu.co/bitstream/10819/5694/1/Revisi\%C3\%B3n_Constructo_Resili encia_Rodriguez_2017.pdf

Suárez, A. (2009). La masacre del salado: esa guerra no era nuestra. https://bit.ly/3v4YY9c

Unidad para la Atención y la Reparación Integral a las Víctimas (2021). Red Nacional de InformaciónRNI. https://www.unidadvictimas.gov.co/es/direccion-de-registro-y-gestion-de-lainformacion/red-nacional-de-informacion-rni/37825

Suarez, A. (2002). Resiliencia descubriendo las propias fortalezas. Paidós.

Taylor, M. (2011). Viviendo en "aquellos tiempos" en Ixcán, Guatemala: la violencia y la vida en las PAC. Revista del Centro de Investigaciones Regionales de Mesoamérica, 32 (53), 157-188.

Terzi, S. (2013). Secure attachment style, coping with stress and resilience among university students. The Journal of Happiness and Well-Being, 1(2), 97-109.

Theis, J. (2007). The Eye of Argon. Wildside Press, LLC

Unidad para la Atención y Reparación Integral a las Víctimas (2010). Indice de riesgo de victimización 2010-2014. Autor. https://issuu.com/iovasernadiseno/docs/libro_irv 
Candanoza-Henríquez., A., E., \& Rojo-Gutiérrez., M., A.

Vásquez, T. (2011). Recursos, política, territorios y conflicto armado. En J. A. Restrepo, T. Vásquez y A. R. Vargas (Eds.). Una vieja guerra en un nuevo contexto. Conflicto y territorio en el Sur de Colombia (pp. 367-428). Universidad Javeriana/ CERAC/ODECOFI.

Wilches Flórez, Á. (2010). La biotecnología en un mundo globalizado. Revista Colombiana de Bioética, 5 (2), 164-169. 